\title{
¿Por qué los beneficiarios de la Leche Liconsa abandonan el programa? Análisis de su satisfacción y percepción de inseguridad en el estado de Colima en 2018
}

Renato F. González Sánchez*

\section{RESUMEN}

Esta investigación busca esclarecer las causas de la deserción de beneficiarias(os) del programa de abasto de leche Liconsa en el estado de Colima. En su diseño y operación, los operadores de Liconsa han cuidado la focalización y su delimitación temporal en los beneficiarios, así como su supervisión, certificación y evaluación. Estudios previos han presentado evidencia de los positivos impactos nutricionales, económicos y de la satisfacción de los beneficiarios del programa. Con base en una muestra estadística de 451 personas (201 ex beneficiarios y 250 beneficiarios) y, se determinó que las percepciones de inconveniencia por inseguridad o los horarios de reparto, la baja satisfacción por precio y calidad de la leche, y las condiciones del local de reparto, entre otros aspectos, hacen que los beneficiarios del programa desistan de continuar en el mismo.

Palabras clave: evaluación de programas sociales, análisis de componentes principales, modelo de regresión biprobit.

Clasificación JEL: C3, D91, H490.

* Profesor investigador de tiempo completo en la Facultad de Economía, Universidad de Colima, México. Correo electrónico: refrgosa@ucol.mx 


\section{ABSTRACT \\ Why do Liconsa's Beneficiaries Abandon the Program? An Analysis of Liconsa Beneficiaries' Satisfaction and Insecurity Perception in Colima, Mexico, 2018}

This paper explains the causes of beneficiaries' desertion of the milk distribution federal program of Liconsa in the state of Colima, Mexico. Liconsa's design and implementation met stringent regulations on focalization, time delimitation, and it is regularly supervised and evaluated. Previous studies provide evidence of positive impacts on children nutrition, economic contribution to families' food expenditures and beneficiaries' satisfaction with the program. Based on a sample of 451 Liconsa recipients (and former recipients) and several statistical methods, it was demonstrated that the perception of insecurity and inconvenience related to delivery schedule, as well as a low satisfaction related to milk's price and quality, have a significative influence in abandoning the program by the beneficiaries in Colima.

Keywords:social program evaluation, principal component analysis, biprobit regression model.

JEL Classification:C3, D91, H490.

\section{INTRODUCCIÓN}

El Programa de Abasto Social de Leche Liconsa (PASL) es uno de los programas más longevos de la historia de México. Mientras que muchos programas (de otras secretarías) cambiaron o desaparecieron, generalmente como resultado de la modificación de los modelos de crecimiento del país, el programa de Liconsa (con otros nombres) ha operado de manera continua desde 1944 hasta el presente, incluso se ha ampliado geográficamente y en objetivos programáticos (BravoFlores, 2019). Actualmente es uno de los programas de política social más regulados, supervisados y evaluados (Soto-Romero et al., 2004), en virtud de su naturaleza e impacto social, generalmente asociado a la nutrición (Shamah et al., 2007), así como a la satisfacción sobre el producto y servicio por los usuarios (Cogco et al., 2013).

Lo anterior implica una clara focalización del programa en aspectos socioeconómicos, orientado a personas de bajos ingresos y dirigido a infantes, ancianos y mujeres de ciertas edades, aunque este elemento 
fue agregado recientemente. Por tanto, el programa de abasto de leche es un modelo para la operación de otros programas sociales: resuelve un problema de mercado, está focalizado, su operación es temporal (desde la perspectiva de los beneficiarios, pues sólo aplica a niños y ancianos) y su impacto es comprobable.

Es posible que su cobertura en el país haya aumentado considerablemente dadas sus características y operación en el marco de diversos programas sociales (Prospera, Programa de Apoyo Alimentario, Programa de Abasto Rural, Comedores Comunitarios, Programa de Apoyo a la Educación Indígena, Desayunos Escolares, Pensión para Adultos Mayores). Entre 2000 y 2017 la cobertura de beneficiarios del programa Liconsa creció a una tasa anual promedio de $2.2 \%$ en Colima, mientras que a nivel nacional fue de $2.7 \%$. Esto implicó que en estos 17 años los beneficiarios se duplicaran hasta 6.4 millones.

A pesar de estas virtudes, cabe preguntarse ¿por qué ciertos derechohabientes deciden dejar de recibir el apoyo del Programa? En el caso de Colima no sería un problema, pues a finales de 2018 se reportaron 1,675 bajas de un padrón de aproximadamente 30,360 beneficiarios o derechohabientes activos. El problema radica en las auditorías externas al PASL, donde uno de los indicadores es la cobertura; no cubrir a la población vulnerable, implica observaciones a la administración. En este sentido, para este trabajo se instrumentó un grupo de enfoque con la administración y promotores del PASL en Colima, y se llegó a la conclusión de que los motivos que propician la salida de los derechohabientes son los temores ligados a la inseguridad y las inconveniencias por el horario de atención en las lecherías. Como es evidente, estos problemas no son variables que el PASL pueda modificar. De 2016 a 2018, la inseguridad se vuelve un problema serio en la entidad. ${ }^{1}$ Estos elementos se convierten en las hipótesis de trabajo para esta investigación. Con un cuestionario se crean variables para las hipótesis y se evalúa la conveniencia y satisfacción de los beneficiarios. Los resultados se comparan con los obtenidos de las personas que decidieron dejar el programa.

\footnotetext{
De acuerdo con datos del Secretariado Ejecutivo del Sistema Nacional de Seguridad Pública (SESNSP), entre 2016 y 2018 en el Estado de Colima hubo 105.8 y 94.5 homicidios por cada 100 mil habitantes. Esto convirtió a la entidad en la más violenta del país.
} 
El trabajo se divide en tres apartados además de esta introducción. En el primero se revisan algunos elementos de la evaluación de programas sociales y los estudios similares al presente. En el segundo se discute el cuestionario, el esquema de muestreo, algunas características socioeconómicas de la muestra y los métodos estadísticos empleados. En el tercero se presenta la evidencia empírica (basada en análisis estadísticos) que responde a la pregunta de investigación. Finalmente se exponen las conclusiones y recomendaciones.

\section{REVISIÓN DE BIBLIOGRAFÍA}

Los gobiernos deciden implementar programas sociales por diversas razones, como reducir la pobreza y vulnerabilidad, construir capital humano, mejorar las condiciones de vida, entre otros. En este tenor, pueden verse como inversión en capital humano para mejorar la acumulación de activos productivos y romper la transmisión generacional de pobreza, protección contra choques e inversión para reducir la pobreza (Barrientos y Hulme, 2009)

Gonzalez-Tiburcio (2001) considera que los programas sociales buscan maximizar el impacto social y la eficiencia interna de su operación. Por eso cobra sentido que los programas sean evaluados en su impacto social y en la manera en cómo fueron implementados (denominada de operación o proceso). El autor también afirma que la evaluación es demandada por la sociedad civil en cuanto a la transparencia del gasto público y rendición de cuentas, así como por la administración pública, con miras a operar programas de manera más eficaz y eficiente.

La evaluación de la satisfacción del usuario (o beneficiario) de algún programa social tiene importancia por las razones siguientes: 1) "La percepción de los usuarios puede servir para poner en perspectiva la información oficial. [...] Debe considerarse la discordancia entre las percepciones de los usuarios y los registros, pues es una alerta de posibles deficiencias de los segundos". 2) "La visión que tienen los usuarios puede señalar problemas que los sistemas objetivo de monitoreo pasan por alto, con lo que facilitan la identificación de áreas a mejorar, las acciones a realizar y los recursos a emplear" (Lobato-Calleros et al., 2016: 31-32). 3) En ocasiones, algunos programas sociales presentan dificultades para detectar y mejorar ciertos servicios del gobierno, por 
lo cual la evaluación de la satisfacción de los beneficiarios es una buena aproximación para valorar los resultados (Kelly y Swindell, 2002).

Existen diversos estudios que evalúan al PASL, así como la satisfacción de sus beneficiarios. Por ejemplo, Soto-Romero et al. (2004) coordinaron la evaluación del Programa con una amplia metodología que incluyó: a) gestión y apego a Reglas de operación, b) cobertura, c) costos, beneficios y resultados, y d) impacto. En este último, además del nutricional, por primera ocasión se evaluó la satisfacción del usuario. De esta manera, los autores encontraron que el programa de leche fortificada evita problemas de desnutrición, anemia y pérdida de peso entre la población beneficiada; y en cuanto a lo económico, la leche a un precio menor que la del mercado, implica un ahorro en consumo de aproximadamente 1,500 pesos al año por familia.

León-Flores (2010) y Lobato-Calleros et al. (2016) evaluaron la satisfacción de los beneficiarios de leche Liconsa en sus presentaciones en polvo y líquida (la diferencia entre ambas es que la primera es para zonas rurales, y la segunda, urbanas; además, la manera de operar el programa en cada caso es diferente). Las metodologías se basan en el índice mexicano de satisfacción de usuarios (IMSU), en una muestra de 2,400 encuestas a nivel nacional, y ambos emplean ecuaciones estructurales. Sus resultados indican que la calidad percibida (producto, atención, etc.), las expectativas y la satisfacción con el programa influyen en la confianza en el mismo, es decir, en la lealtad y recomendación de producto. Este trabajo recopila la metodología y los resultados que los autores emplearon para evaluar la satisfacción de beneficiarios de diferentes programas de Sedesol publicados en 2006, 2007 y 2011.

Por su parte, Cogco et al. (2013) desarrollaron una metodología denominada "Modelo de satisfacción de los beneficiarios de los programas sociales" (IMSAB). Su muestra fue de 1,060 encuestas en 53 localidades de 10 estados del país, para lo cual emplearon ecuaciones estructurales. Sus resultados indican que la calidad del beneficio influye positiva y significativamente en la satisfacción del beneficiario. En menor medida, la gestión del beneficio, la cohesión social y la contraprestación influyen también en la satisfacción. Los autores esperaban que esta última variable influyera adversamente en la satisfacción, pero los beneficiarios no la calificaron negativamente. En cambio, las expectativas y la imagen del programa no influyen en la satisfacción. 
Existen algunos trabajos que evalúan el impacto nutricional de la leche Liconsa. Por ejemplo, Shamah et al. afirman que: "Los resultados indican una alta efectividad de la leche fortificada para disminuir las tasas de anemia y deficiencia de hierro" (2007: 252), de ahí el efecto positivo en la salud de miles de niños mexicanos, cuyas familias son beneficiarias del programa. Por su parte, Grijalvo-Haro et al., a través de un cuasi-experimento, muestran que "el consumo de esta leche beneficia el estado bioquímico de hierro y zinc en los niños" (2014: 331), por lo que es mejor, comparada con la leche comercial.

Finalmente, Bravo-Flores (2019) estudia las causas del abandono de los beneficiarios de PASL en Colima, y las relacionan con la percepción de inseguridad, así como algunos aspectos socioeconómicos de las personas que dejan el servicio. Este trabajo destaca porque presenta una propuesta de acciones de mejora de la administración de la procesadora de leche de Liconsa en el estado, así como para operación del programa.

\section{Metodología}

\subsection{La encuesta y el diseño de muestreo}

Para el esquema se consideró como universo muestral las personas que hasta agosto de 2018 se habían dado de baja o habían dejado de recoger la leche (Cuadro 1). En total, y después de excluir al municipio de Ixtlahuacán por baja representatividad, se obtuvo 1,655 exbeneficiarios. A partir de la aplicación de una encuesta piloto (10 cuestionarios en 9 municipios), y considerando como variable aleatoria los días que las personas trabajaron la semana previa a la aplicación de la encuesta, se establece la base de datos para el esquema de muestreo, que en este caso es el estratificado aleatorio. Esta variable se consideró debido a que a las personas con bajos niveles de ingreso generalmente se dificulta encontrar trabajo; este tipo de personas son beneficiarias de los programas sociales de Sedesol. Con base en Montesinos et al. (2010), se aplicó la fórmula:

$$
n=\frac{N(z \alpha)^{2} \sum_{h=1}^{E} W_{h} S_{h}^{2}}{N d^{2}+\left(Z_{\alpha}\right)^{2} \sum_{h=1}^{E} W_{h} S_{h}^{2}},
$$


Donde $\mathrm{N}$ (población) $=1,655 ;\left(\mathrm{z}_{\alpha / 2}\right)^{2}=(1.96)^{2}=3.841 ; \mathrm{d}=$ (precisión* promedio estratificado) $=0.086 * 4.1=0.352 ; \sum_{(h=l)}^{E} W_{h} S_{h}^{2}=$ 6.39. Con estos datos se tiene un tamaño de muestra de $176.9(\approx 177)$ ex beneficiarios a encuestar. La afijación de la muestra se presenta también en el Cuadro 1

\section{CUADRO 1}

RESUMEN DE RESULTADOS DE MUESTREO ESTRATIFICADO

\begin{tabular}{|c|c|c|c|c|c|c|c|c|c|}
\hline Estratos & $\mathrm{Nj}$ & $\begin{array}{c}\text { Prom. } \\
* /\end{array}$ & $S_{h} 2 * I$ & $\begin{array}{l}W h= \\
N j / N\end{array}$ & $W \mathrm{~W}^{*} \mathrm{~S}^{2}{ }_{h}$ & $\begin{array}{c}\text { Afijación } \\
\text { de } \\
\text { muestra }\end{array}$ & $\begin{array}{c}\text { Casas } \\
\text { visitadas }\end{array}$ & $\begin{array}{c}\text { Útiles } \\
\text { para } \\
\text { análisis }\end{array}$ & $\begin{array}{c}\text { Muestra } \\
\text { complementaria** }\end{array}$ \\
\hline Armería & 148 & 3.1 & 7.88 & 0.09 & 0.70 & 16 & 28 & 0 & 17 \\
\hline Colima & 193 & 3.6 & 7.21 & 0.12 & 0.84 & 21 & 49 & 36 & 38 \\
\hline Comala & 114 & 2.9 & 8.22 & 0.07 & 0.57 & 12 & 49 & 40 & 21 \\
\hline Coquimatlán & 94 & 3.0 & 2.49 & 0.06 & 0.14 & 10 & 21 & 18 & 20 \\
\hline Cuauhtémoc & 41 & 5.4 & 6.32 & 0.02 & 0.16 & 4 & 27 & 23 & 25 \\
\hline Manzanillo & 518 & 4.1 & 8.32 & 0.31 & 2.60 & 55 & 64 & 31 & 60 \\
\hline Minatitlán & 110 & 4.9 & 6.71 & 0.07 & 0.45 & 12 & 42 & 0 & 41 \\
\hline Tecomán & 414 & 4.1 & 3.66 & 0.25 & 0.91 & 44 & 57 & 15 & 23 \\
\hline V. Álvarez & 23 & 5.5 & 1.17 & 0.01 & 0.02 & 2 & 15 & 14 & 29 \\
\hline $\mathrm{N}$ & 1,655 & & & & 6.39 & 177 & 352 & 177 & 274 \\
\hline
\end{tabular}

Fuente: elaboración propia.

Notas: */ Promedio y varianza por municipio. Son valores obtenidos de una muestra piloto de 10 encuestas a cada municipio con valores de días a la semana que los encuestados trabajaron. $* *$ / La muestra complementaria son las encuestas aplicadas a beneficiarios activos del programa. Los derechohabientes de Ixtlahuacán (15), se excluyeron por poca representatividad, inseguridad o lejanía.

Debe notarse que en la base de datos de ex derechohabientes se contaba con la dirección, y por eso la encuesta se aplicó en los hogares. Sin embargo, fue algo común que no se encontrara a las personas, por razones como que "se cambió de casa", "nadie lo conoce", o simplemente no había nadie en el domicilio al pasar. Asimismo, también se encontraron problemas de seguridad, en el sentido de que había personas en la calle que inquirían a los encuestadores sobre sus motivos. Los encuestadores, en la mayoría de los casos, prefirieron no insistir en la búsqueda de dichos ex beneficiarios. Estos problemas de seguridad 
fueron más acentuadosse acentuaron en las colonias urbanas de Manzanillo y Tecomán. Ello significó que realmente se buscara a un grupo más grande de personas de las que originalmente se había establecido en la muestra. Por otra parte, se decidió no aplicar los cuestionarios a no beneficiarios de los municipios de Armería y Minatitlán, debido a que ahí el programa vende leche en polvo, y el cuestionario no fue diseñado para este tipo de leche, sino la líquida. Finalmente, para tener una muestra "espejo" complementaria, se aplicó el mismo cuestionario instrumento a los beneficiarios activos en las lecherías de los mismos municipios. El levantamiento de encuestas se realizó entre diciembre de 2018 y enero de 2019.

El cuestionario se divide en tres apartados. Uno de ellos es la parte socioeconómica de los encuestados, donde se les pregunta su nivel de estudios, situación marital y laboral, así como edad y años de beneficiario, entre otros. Otra parte cuestionadel cuestionario pregunta sobre su percepción de riesgo al dirigirse recoger la leche en al punto de venta de la leche. Estas percepciones se recogen mediante una escala Likert del 1 al 5. Otro apartado colecta la percepción de la satisfacción con servicios de Liconsa, nuevamente en escala Likert. El Cuadro 2 muestra algunas características socioeconómicas de la muestra aplicada. Debe observarse que de estas variables, sólo existe relación entre el nivel educativo y la situación laboral (prob $\left.\left[\chi_{6 \mathrm{gl}}^{2}=20.682\right]=0.002\right)$, en el sentido de que al incrementarse la escolaridad, las personas pasan de estar desocupadas, o no percibir salario, a tener un trabajo asalariado $\mathrm{o}$ actividades de autoempleo ( $\mathrm{tau} \mathrm{b}$ de Kendall $=0.184$, prob $=0.000$ ). En particular, al realizar un análisis de correspondencia, se demuestra que existe una alta cercanía entre la situación de que el encuestado esté desocupado o jubilado y el nivel de estudios de primaria (inercia de $4.5 \%)$. 


\section{CUADRO 2}

CARACTERÍSTICAS SOCIOECONÓMICAS DE LOS ENCUESTADOS

\begin{tabular}{|c|c|c|c|c|}
\hline Variable & Indicador & Ex beneficiario & $\begin{array}{l}\text { Derecho } \\
\text { habiente }\end{array}$ & Subtotal \\
\hline \multirow{4}{*}{ Nivel educativo } & Bachillerato /Universitario* & 30 & 54 & 84 \\
\hline & Primaria & 54 & 99 & 153 \\
\hline & Secundaria & 83 & 110 & 193 \\
\hline & Sin estudios & 10 & 11 & 21 \\
\hline \multirow{4}{*}{ Situación marital } & Casado(a) & 125 & 186 & 311 \\
\hline & Separado(a), Divorciado (a) & 16 & 38 & 54 \\
\hline & Soltero(a) & 24 & 30 & 54 \\
\hline & Viudo(a) & 12 & 20 & 32 \\
\hline \multirow{3}{*}{ Situación laboral } & Desocupado o jubilado & 46 & 94 & 140 \\
\hline & Trabaja sin salario & 24 & 33 & 57 \\
\hline & $\begin{array}{l}\text { Tiene trabajo o negocio de } \\
\text { autoempleo }\end{array}$ & 107 & 147 & 254 \\
\hline \multirow{4}{*}{$\begin{array}{c}\text { Si no pudo ir a Liconsa, ¿qué } \\
\text { hace regularmente? }\end{array}$} & $\begin{array}{l}\text { Compra otra leche en otro } \\
\text { lado }\end{array}$ & 99 & 94 & 193 \\
\hline & $\begin{array}{l}\text { Les hace otra bebida a los } \\
\text { niños }\end{array}$ & 21 & 36 & 57 \\
\hline & $\begin{array}{c}\text { Se espera a comprarla otro } \\
\text { día en Liconsa }\end{array}$ & 57 & 144 & 201 \\
\hline & Total general & 177 & 274 & 451 \\
\hline
\end{tabular}

Fuente: elaboración propia con base en datos de 451 encuestas.

Notas* / Se agregaron los datos de bachillerato y licenciatura porque representan $2.4 \%$ del nivel educativo de la muestra.

\subsection{Métodos estadísticos empleados}

En esta investigación los métodos empleados fueron: el análisis de componentes principales categóricos (ACPC). Los resultados son comparados con métodos no paramétricos, así como en el modelo de regresión aparentemente no relacionado biprobit recursivo. La selección de los métodos está acorde con las hipótesis de la investigación, así como también con la naturaleza de los datos. 
El ACP es una técnica de reducción de dimensiones que, como indica Santos Peña et al. "condensa un conjunto de $K$ variables originales en otro conjunto $W$ de variables intrínsecas o factores, con $W<K$; los W factores son una combinación lineal de K" (2004: 455). Debe notarse que las variables originales generalmente están correlacionadas, como el caso de las Likert (con valores ordinales de 1 a 5), que en este trabajo expresan la percepción sobre inseguridad, inconveniencia o satisfacción para recoger la leche. Para el procedimiento, se empleó la matriz de correlaciones policórica de las variables originales, dado que se consideró que estas variables no son normales, son categóricas (no son continuas) y posiblemente presenten alta curtosis (Muthén y Kaplan, 1992); en este sentido, se aplica el ACP categórico. Además, se rotaron los ejes con un método ortogonal (que implica que los factores de regresión derivados se producen sin correlación entre ellos) denominado varimax (Field, 2009: 644). Se utilizó el Programa Factor(R) para estas estimaciones.

En el ACP se usan diferentes estadísticos que pueden ayudar a ajustar mejor el resultado de agrupación de las variables originales. Entre éstos están la medida de adecuación de la muestra Kaiser-MeyerOlkin (KMO), el cual toma valores entre 0 y 1 . Los valores cercanos a 0 indican que las variables originales tienen poco en común entre ellas. La prueba de esfericidad de Barttlet examina si la matriz de correlación debería factorizarse, o sea, si los datos no son independientes. Esta es una prueba de ji-cuadrada con una prueba estadística, que es una función del determinante de la matriz de correlación de variables. Se espera una significancia mayor a 5\% (Hair et al., 1999).

La manera de determinar qué variables originales son una combinación lineal de una variable intrínseca es a través de las cargas factoriales, así como también por el valor de la comunalidad. Se recomienda tomar los valores relativamente más altos de las cargas factoriales (por arriba de 0.5 ); mientras que los valores bajos de la comunalidad indican que sus variables originales no son parte de ningún agrupamiento. Finalmente, se emplea el estadístico de alfa de Cronbach para determinar la confiabilidad de las variables originales agrupadas (Hair et al., 1999). 


\subsection{Modelo de regresión aparentemente no relacionada (SUR, por sus siglas en inglés) biprobit recursivo}

Este modelo integra dos ecuaciones simultáneas, con variables dependientes dicótomas, relacionadas a nivel de dependencia. El modelo es adecuado para estimar el efecto de una variable endógena binaria $\mathrm{W}$ sobre otra variable dicótoma y. Su expresión matemática es:

$$
\begin{aligned}
& W^{*}=x^{\prime 1} \beta_{1}+\varepsilon_{1}, W=1 \text { si } W^{*}>0, \mathrm{y} 0 \text { de otra manera } \\
& y^{*}=x^{\prime 2} \beta_{2}+\gamma W+\varepsilon_{2}, y=1 \text { si } y^{*}>0, \mathrm{y} 0 \text { de otra manera }
\end{aligned}
$$

Además, se supone que $\left(\begin{array}{c}\varepsilon_{1} \\ \varepsilon_{2}\end{array} x_{1}, x_{2}\right) \sim N\left[\left(\begin{array}{ll}0 \\ 0\end{array}\right),\left(\begin{array}{ll}1 & \rho \\ 0 & 1\end{array}\right)\right]$, donde $\rho($ rho $)$ es la correlación tetracórica entre los residuos conjuntamente estimados de $y \wedge \mathrm{W}$. Ésta es de especial atención, dado que se establece un estadístico de prueba (Wald o Multiplicador de Langrange) para demostrar que $\rho \neq 0$. Si esto no se prueba, entonces las ecuaciones $y^{*}$ y $W^{*}$ pueden estimarse por separado.

Dado que la variable $\mathrm{W}$ aparece en la parte derecha de la segunda ecuación, se trata de un modelo recursivo de ecuaciones simultáneas. De acuerdo con Greene (2012: 746), la "naturaleza endógena de una de las ecuaciones en el lado derecho de la otra ecuación, puede ser ignorada, en la construcción de la función log-verosimilitud" (2012: 746). La distribución conjunta de $(W, y)$ (condicionado por $\left.\mathrm{x}_{1}^{\prime} \mathrm{y} \mathrm{x}_{2}^{\prime}\right)$ tiene cuatro elementos (basado en Winkelmann, 2011):

$$
\begin{array}{l|l}
\mathrm{P}(\mathrm{y}=0, \mathrm{~W}=0 & \left.\mathrm{x}_{2}^{\prime}, \mathrm{x}_{1}^{\prime}\right)=\mathrm{P}\left(\varepsilon_{2} \leq-x_{2}{ }_{2} \beta_{2}, \varepsilon_{1} \leq-x_{1} \beta_{1}\right) \\
\mathrm{P}(\mathrm{y}=1, \mathrm{~W}=0 & \left.\mathrm{x}_{2}^{\prime}{ }_{2}, \mathrm{x}_{1}{ }_{1}\right)=\mathrm{P}\left(\varepsilon_{2} \leq-x_{2}{ }_{2} \beta_{2}, \varepsilon_{1} \leq-x_{1}^{\prime} \beta_{1}\right) \\
\mathrm{P}(\mathrm{y}=0, \mathrm{~W}=1 & \left.\mathrm{x}_{2}^{\prime}, \mathrm{x}_{1}^{\prime}\right)=\mathrm{P}\left(\varepsilon_{2} \leq-x_{2}{ }_{2} \beta_{2}, \gamma, \varepsilon_{1}>-x_{1}{ }_{1} \beta_{1}\right) \\
\mathrm{P}(\mathrm{y}=1, \mathrm{~W}=1 & \left.\mathrm{x}_{2}^{\prime}{ }_{2}, \mathrm{x}_{1}^{\prime}\right)=\mathrm{P}\left(\varepsilon_{2} \leq-x_{2}^{\prime}{ }_{2} \beta_{2}, \gamma, \varepsilon_{1}>-x_{1}^{\prime} \beta_{1}\right)
\end{array}
$$

Con esta base, Greene establece la función log verosimilitud, a partir de la cual se obtienen los estimadores de máxima verosimilitud (2012: 739).

$$
\begin{aligned}
& \ln L=\sum_{i=l}^{n} \ln \Phi_{2}\left(w_{i 1}, w_{i 2}, \rho_{i}\right) ; \text { donde } w_{i j}=q_{i j} z_{i j}, z_{i j}=x_{i j}^{\prime} \beta_{j} j=1,2, \rho_{i^{*}}= \\
& q_{i 1} q_{i 2} \rho, \\
& q_{i 1}=2 y_{i 1}-1, q_{i 2}=2 y_{i 2}-1 \text {. Así } q_{i j}=1 \text {, si } \mathrm{y}_{\mathrm{ij}}=1, \mathrm{y} q_{i j}=-1 \text {, si } \mathrm{y}_{\mathrm{ij}}=0 .
\end{aligned}
$$


Para el caso del estudio aquí propuesto, $W=1$ denota si el encuestado ya no es beneficiario del PASL en el momento de la aplicación del cuestionario (esta variable es potencialmente endógena); mientras que $\mathrm{y}=$ 1 define al entrevistado que afirma que deja el programa por problemas de inseguridad.

\subsection{Comparación de mediana de muestras con métodos no paramétricos}

La finalidad de emplear estas técnicas estadísticas es determinar si una variable es semejante en dos o más muestras. Una manera de hacer esto es probando si dicha variable tiene el mismo promedio, o de manera alternativa, su mediana. En este trabajo se busca saber si entre las muestras de beneficiarios activos y exderechohabientes, o por alguna característica socioeconómica, existen variables con estas características. Si, por ejemplo, las variables bajo análisis se distribuyen como normales y presentan varianzas homogéneas en cada muestra (de beneficiarios o no), entonces se pueden emplear las pruebas de t-Student, o bien el análisis de varianza. Si estas restricciones no se cumplen, entonces deben emplearse las pruebas no paramétricas; en este trabajo se utiliza Mann-Whitney (U-MW), técnica no paramétrica para dos poblaciones usando muestras independientes cuando la escala es al menos ordinal (Infante y Zarate, 2000: 537). Si se tienen tres o más muestras independientes con las características descritas, entonces se aplica en estadístico de Kruskal Wallis.

Antes de emplear este método, las variables son analizadas para determinar si se acercan a una distribución normal. Para esto se emplean las pruebas de Kolmogorov-Smirnov, Shapiro-Wilks y Shapiro-Francia. Éstas se tienen en diferentes programas estadísticos. En este caso se empleó el de Stata para las pruebas de normalidad y el SPSS para la prueba de U-MW.

\section{ESTIMACIÓN Y ANÁLISIS DE RESULTADOS}

\subsection{Análisis de componentes principales categóricos}

Para este ejercicio estadístico se emplearon dos bases de datos del cuestionario. La primera se refiere a las variables de percepción de riesgo al recoger la leche en el punto de venta, con escala Likert del 1 al 5, 
donde 1 es equivalente a que el encuestado está "completamente en desacuerdo" y 5 significa "completamente de acuerdo", para las afirmaciones establecidas.

En cada caso se emplea la matriz de correlaciones policórica de las variables originales, aspecto que se recomienda por el tipo de variables (categóricas) y por los valores de varianza y curtosis. Los estadísticos más importantes para cada matriz se presentan en el pie de cada cuadro. En los dos ejercicios se rotaron los ejes con una técnica ortogonal (varimax), por lo que valores factoriales resultantes no presentan correlación entre sí.

Como se observa en el Cuadro 3, a partir de 10 variables originales se obtienen dos variables intrínsecas que mantienen $51.6 \%$ de la varianza. El valor de cargas factoriales fue establecido para ser mayor de 0.5 , de manera que existiera una mejor agrupación de variables. Aunque se presentaron algunos valores de baja comunalidad para algunas variables observables, los valores de cargas factoriales indicaron que no era necesario excluir variables. Los indicadores del determinante de la matriz de correlaciones como el KMO y la prueba de Barttlet indican que esta matriz puede factorizarse adecuadamente. Debe notarse, sin embargo, que los valores de Alfa de Cronbach indican una asociación adecuada entre las variables agrupadas. En este sentido, se propone el siguiente renombramiento para las variables intrínsecas:

INSEG $=$ Percepción de inseguridad por la distancia, el camino oscuro y/o inseguro, soledad y dinero insuficiente;

$\mathrm{INCONV}=$ Percepción de inconveniencia por horario (del trabajo o escolar), trato de distribuidor. 


\section{CUADRO 3}

ACP PARA PERCEPCIÓN DE INSEGURIDAD E INCONVENIENCIA CON EL SERVICIO DEL PASL

\begin{tabular}{|c|c|c|c|c|c|}
\hline Variable & INSEG & INCONV & Com & Varianza & Curtosis \\
\hline $\begin{array}{l}\text { No quiero ir por la leche por } \\
\text { el inconveniente del horario } \\
\text { temprano }\end{array}$ & & 0.632 & 0.501 & 1.823 & -1.127 \\
\hline $\begin{array}{l}\text { En ocasiones tengo flojera de } \\
\text { levantarme temprano para ir por } \\
\text { la leche }\end{array}$ & & 0.547 & 0.386 & 1.686 & -1.234 \\
\hline $\begin{array}{l}\text { Los horarios para ir al trabajo no } \\
\text { me dejan ir por la leche }\end{array}$ & & 0.783 & 0.619 & 1.814 & -1.157 \\
\hline $\begin{array}{l}\text { Los horarios de la escuela de mis } \\
\text { hijos no me permiten recoger } \\
\text { la leche }\end{array}$ & & 0.726 & 0.582 & 1.642 & -0.873 \\
\hline $\begin{array}{c}\text { El trato de la persona que } \\
\text { distribuye me desanima para ir } \\
\text { por la leche }\end{array}$ & & 0.621 & 0.408 & 1.432 & -0.456 \\
\hline $\begin{array}{l}\text { No siempre quiero ir por la leche } \\
\text { porque el camino puede ser } \\
\text { inseguro }\end{array}$ & 0.793 & & 0.647 & 1.731 & -1.131 \\
\hline $\begin{array}{l}\text { La distancia de casa a lechería } \\
\text { me desalienta para ir por la leche }\end{array}$ & 0.685 & & 0.54 & 1.493 & -0.653 \\
\hline $\begin{array}{l}\text { El camino está oscuro o han } \\
\text { ocurrido crímenes y tengo miedo } \\
\text { a salir por la leche }\end{array}$ & 0.724 & & 0.581 & 1.672 & -1.082 \\
\hline $\begin{array}{l}\text { Estoy solo(a) y me es difícil ir por } \\
\text { la leche }\end{array}$ & 0.541 & & 0.503 & 1.652 & -0.969 \\
\hline $\begin{array}{c}\text { A veces no tengo todo el dinero } \\
\text { para ir a comprar la leche }\end{array}$ & 0.611 & & 0.392 & 1.942 & -1.309 \\
\hline Autovalor & 2.60 & 2.56 & & & \\
\hline Proporción de la varianza & 0.260 & 0.256 & & & \\
\hline Confiabilidad (Alfa de Cronbach) & 0.788 & 0.786 & & & \\
\hline
\end{tabular}

Fuente: elaboración propia con base en los datos de 451 encuestas.

Notas: $\mathrm{Com}=$ comunalidad. Método de extracción: análisis de componentes principales basado en la matriz de correlación policórica. Los valores de varianza y curtosis mayores a 1 (en valor absoluto) indicaron la necesidad de emplear ACP categórico. Método de rotación: varimax normalizada. Medida Kaiser-Meyer-Olkin de adecuación de muestreo $=0.844$ (buena). Prueba de esfericidad de Bartlett: $($ Chi-cuadrado $=10602.7)$ con Probabilidad. $=0.000$. Determinante $=0.0922$. 
El segundo conjunto de datos se refiere a la satisfacción del servicio proporcionado por Liconsa. Nuevamente se emplea la escala Likert, donde 1 expresa el nivel más bajo de satisfacción y 5 el más alto. Como muestra el Cuadro 4, a partir de 6 variables originales se obtienen 2 variables intrínsecas que mantienen $62.5 \%$ de la varianza. No se excluye ninguna variable original causada por el valor de cargas factoriales (establecido a no menos de 0.5 ) o los valores de la comunalidad. Los indicadores del determinante de la matriz de correlaciones como el KMO y la prueba de Barttlet indican que esta matriz puede factorizarse adecuadamente. Los valores de Alfa de Cronbach indican una asociación adecuada o débilmente confiable entre las que constituyen las nuevas variables intrínsecas. El renombramiento es el siguiente:

SAT1 $=\quad$ Satisfacción con horario, trato de distribuidor y condiciones del local.

SAT2 $=\quad$ Satisfacción con el precio y la calidad de la leche.

CUADRO 4

ACP PARA SATISFACCIÓN CON EL SERVICIO DEL PASL

\begin{tabular}{|c|c|c|c|c|c|}
\hline & SAT1 & SAT2 & Comum & Varianza & Curtosis \\
\hline El horario para recoger la leche & 0.735 & & 0.560 & 0.855 & 0.080 \\
\hline El trato de los distribuidores & 0.728 & & 0.554 & 0.833 & 0.828 \\
\hline $\begin{array}{l}\text { Condiciones como cambio, } \\
\text { colas, etc. }\end{array}$ & 0.749 & & 0.572 & 0.888 & -0.574 \\
\hline $\begin{array}{l}\text { Limpieza del local, manejo del } \\
\text { producto, etc. }\end{array}$ & 0.672 & & 0.567 & 0.467 & 0.060 \\
\hline Precio de la leche & & 0.852 & 0.759 & 0.616 & 1.611 \\
\hline Calidad de la leche & & 0.839 & 0.738 & 0.585 & 0.950 \\
\hline Autovalor & 2.149 & 1.601 & & & \\
\hline Proporcion de la varianza & 0.358 & 0.267 & & & \\
\hline Confiabilidad (Alfa de Cronbach) & 0.714 & 0.61 & & & \\
\hline
\end{tabular}

Fuente: elaboración propia con base en los datos de 451 encuestas.

Notas: Comum = comunalidad. Método de extracción: análisis de componentes principales basado en la matriz de correlación policórica. Los valores de varianza y curtosis mayores a 1 (en valor absoluto) indicaron la necesidad de emplear ACP categórico. Método de rotación: varimax normalizada. Medida Kaiser-Meyer-Olkin de adecuación de muestreo $=0.75822$ (aceptable). Prueba de esfericidad de Bartlett: (Chi-cuadrado $=410.3$ ) con Probabilidad. $=0.000$. Determinante $=0.399$. 
Los resultados del ACP Categórico, es decir, los valores factoriales (factor scores), tienen distintos propósitos, como para el análisis de varianza (o pruebas no paramétricas) o para el análisis de regresión, como se presenta a continuación.

\subsection{Comparación de mediana de poblaciones con métodos no paramétricos}

El propósito es probar si algunas características socioeconómicas de los encuestados (nivel educativo, situación marital o laboral), que son o dejaron de ser derechohabientes de Liconsa, influyen en su percepción de inseguridad, inconveniencia o en la satisfacción por el servicio o el producto (leche). Además, se prueban otras variables como edad, años de beneficiario, días trabajados en la semana anterior a la aplicación de las encuestas, entre otras (Cuadro 5). La razón de emplear métodos no paramétricos se debe a que las variables no se distribuyen como normal y las varianzas no son homogéneas. Para esto se corrieron las pruebas de Kolmogorov-Smirnov y Shapiro Wilks, así como la de Levene (estos estadísticos no se presentan en el trabajo).

El Cuadro 5 brinda una descripción de los beneficiarios activos de Liconsa en comparación con aquellos que abandonaron el servicio. Como se observa, los exderechohabientes muestran un nivel de satisfacción por debajo de los derechohabientes activos, en aspectos como horario, trato y condiciones del punto de distribución, satisfacción con el precio y con la calidad del producto. También, los exbeneficiarios de Liconsa tienen percepciones más altas en cuanto a la inseguridad en el tránsito para recoger la leche. Por otra parte, los beneficiarios en activo tienen más tiempo recibiendo la ayuda, son relativamente mayores en edad y compran menos refrescos (sodas o gaseosas) que los no beneficiarios.

Las personas que trabajan sin percibir salario son quienes reportan los niveles más altos de inconveniencia por inseguridad o por el horario de servicio de los puntos de distribución. También son quienes afirman tener el nivel más bajo en satisfacción por precio y calidad de la leche. Este grupo de personas son quienes tienen un más alto número de familiares que trabajan y aportan al ingreso familiar y quienes más compran refrescos a la semana; si se les compara con los entrevistados que están desocupados o jubilados o cuentan con un empleo o autoempleo. 
¿Por qué los beneficiarios de la leche Liconsa abandonan el programa?... $\lceil$ González Sánchez, R. F.

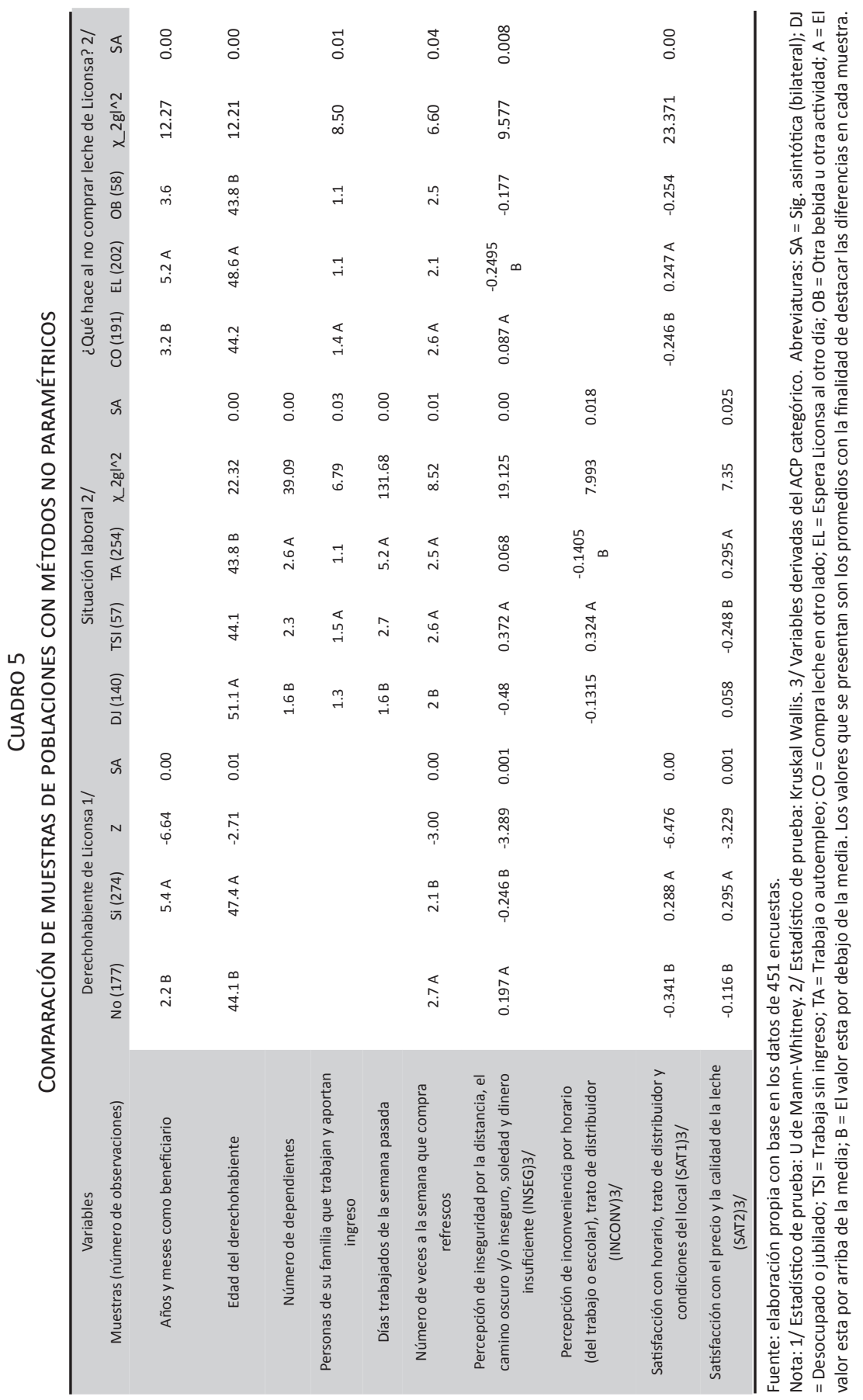


Las personas que afirmaron que esperan al producto de Liconsa en caso de no haber podido recogerlo el día que les corresponde, son quienes tienen la percepción más alta en la satisfacción por el horario, trato del distribuidor y condiciones del local; así como la percepción más baja sobre la inconveniencia por horarios (la comparación es con las familias que deciden comprar la leche en otra tienda o preparar otras bebidas para sus hijos). Este grupo de personas comparativamente tiene más años de beneficiarios y más edad biológica.

\subsection{Modelo de regresión biprobit recursivo}

Como se observa en el Cuadro 6, la prueba estadística del modelo completo (Wald) indica que éste puede emplearse para fines de predicción. Asimismo, el estadístico de prueba para la correlación tetracórica de los residuos de los dos modelos probit (rho) es significativo; por tanto, es más adecuada la estimación conjunta de los modelos.

Como se observa en el Modelo 1 del Cuadro 6, las variables que aumentan la probabilidad de que un derechohabiente de Liconsa deje de serlo son: ser mujer; laborar un mayor número de días a la semana; número de veces que compra refrescos a la semana, o que anteriormente haya abandonado el PASL. Por otra parte, las variables que aumentan la probabilidad de que los beneficiarios se mantengan en el PASL son: nivel medio superior de estudios; estar divorciado (a) o separado (a); familiares derechohabientes a la fecha; alto nivel de satisfacción con los horarios; el trato de distribuidor y las condiciones del local; alta satisfacción con el precio y la calidad de la leche.

Las elasticidades (dy/dx) del Modelo 1 presentadas en el Cuadro 6 indican el porcentaje de cambio en las probabilidades de que abandone el programa. En este sentido, si la derechohabiente es mujer, aumenta $24.3 \%$ la probabilidad; por cada día adicional que haya trabajado la semana anterior a la aplicación de la encuesta, aumenta $2.1 \%$; cada ocasión adicional que compre refrescos a la semana, aumenta 3.6 por ciento.

Si el beneficiario tiene el bachillerato concluido, aumenta $19.2 \%$ la probabilidad de que se mantenga en el programa; si está separado o divorciado, aumenta $26.1 \%$. Por cada familiar que tenga como derechohabiente, aumenta 3.8\%. Al incrementar el nivel de satisfacción (del 1 a 5 en la escala Likert original) con el horario, trato y condiciones del local, la probabilidad de mantenerse en el programa aumenta $16.6 \%$; si 
se trata de la satisfacción con el precio y calidad de la leche, aumenta 8.0 por ciento.

El Modelo 2 del Cuadro 6 indica qué variables aumentan o disminuyen la probabilidad de que el beneficiario deje de serlo por problemas explícitos de seguridad para dirigirse a la tienda por la leche. Este modelo recoge el efecto del Modelo 1, pues la variable dependiente de éste es explicativa para el Modelo 2. Entre las variables que aumentan la probabilidad de observar problemas de seguridad para recoger la leche, están la edad del beneficiario, el aumento del número de dependientes, que ya no sea beneficiario de PASL, que prefiera preparar otras bebidas en lugar de emplear la leche de Liconsa, así como las altas percepciones de inconveniencia por los horarios o de inseguridad en el trayecto para recoger la leche. Las variables que disminuyen la probabilidad de que abandone el programa por problemas de seguridad son su situación laboral (que trabaje sin ingreso o se autoemplee) y que tenga más familiares derechohabientes. Estas últimas variables son contraintuitivas.

Al evaluar las elasticidades, se observa que si el entrevistado ya no es beneficiario de Liconsa aumenta la probabilidad 3.6\% de que haya abandonado el programa por problemas de seguridad. Por cada año adicional de edad, la probabilidad de salir del programa por problemas de inseguridad aumenta $0.02 \%$ y por cada dependiente adicional, $2.6 \%$. $\mathrm{Si}$ el encuestado prefiere hacer una bebida diferente debido a que no pudo comprar leche Liconsa, la probabilidad de salida aumenta por inseguridad $7.9 \%$. Si el encuestado incrementa su percepción de inconveniencia por el horario (cada punto en la escala Likert descrita anteriormente), aumenta $3.7 \%$ la probabilidad de abandonar el servicio por inseguridad; y por cada nivel que aumenta en su percepción de inconveniencia por camino inseguro, aumenta $9.1 \%$ la probabilidad. 


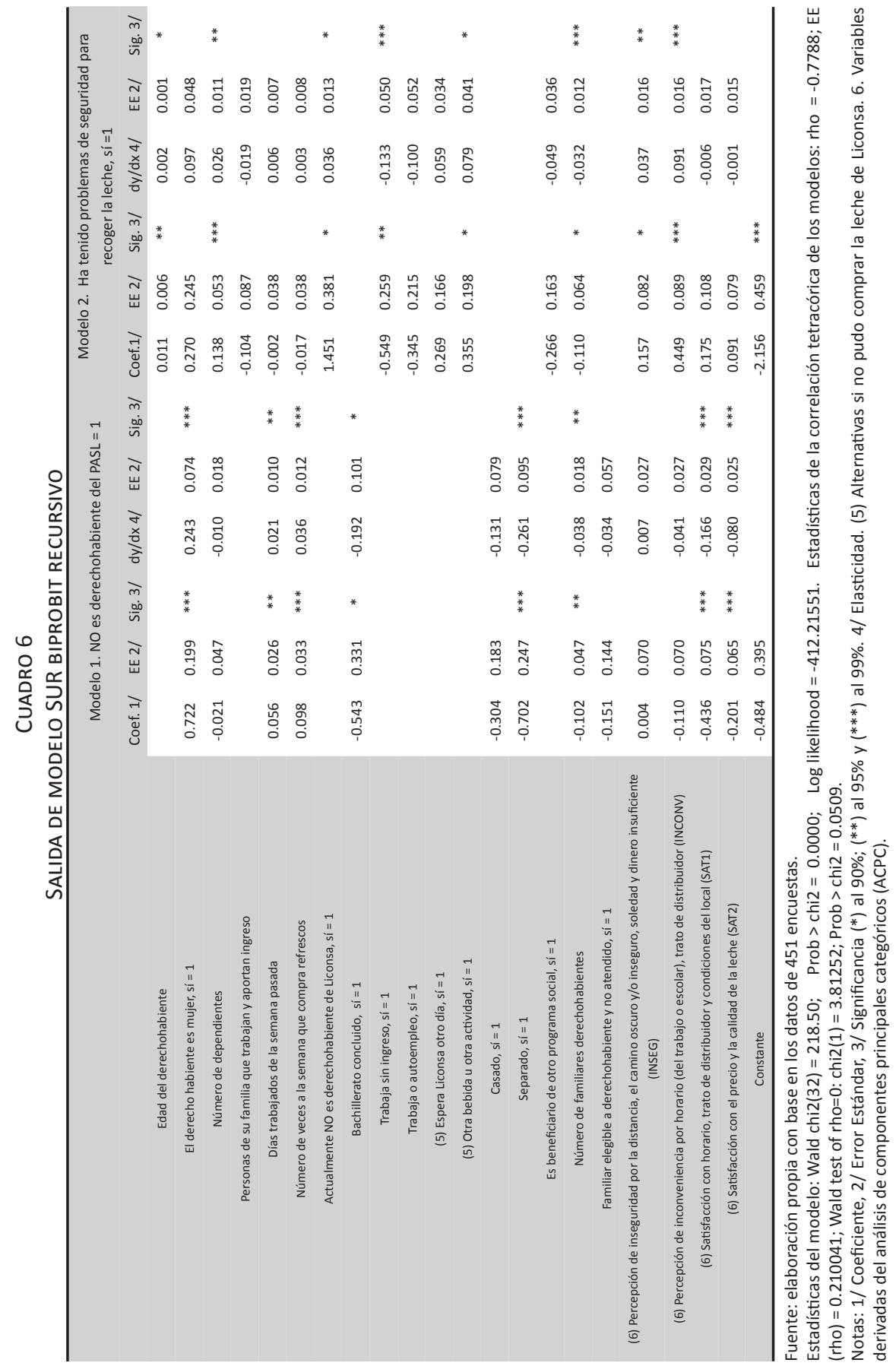




\section{CONCLUSIONES}

Este trabajo surgió de la necesidad de explicar las causas de la deserción de beneficiarios del PASL en Colima por parte de la administración del programa del estado. Si bien se intuía que la inseguridad influía en esta situación, no se contaba con la total certeza sobre ello o de si existían otros elementos que causaban el mismo efecto. Este trabajo se distingue de otros estudios del PASL en el sentido de que los revisados evalúan el impacto, operación o satisfacción del servicio del PASL; mientras que en el nuestro se incorpora la variable "percepción de inseguridad" a la evaluación de la satisfacción por el usuario o beneficiario del programa.

Los resultados indican que la alta satisfacción del programa (por el servicio y el producto) aumenta la retención de los beneficiarios, lo que confirma las aportaciones de León-Flores (2010) y Lobato-Calleros et al. (2016). Por otra parte, las inconveniencias (por horarios o distancias) en que incurren los beneficiarios para recoger la leche líquida aumentan la probabilidad de que abandonen el programa por problemas específicos de inseguridad. Estos resultados se asemejan a lo obtenido por Bravo-Flores (2019). Por otra parte, existen otros elementos que alejan a los beneficiarios del programa, el ser mujer quien recoja el producto, algunos aspectos de comportamiento (sustitución de leche por otras bebidas), el nivel educativo o tipo de empleo, como se discutió en esta investigación.

Lo que no dicen las encuestas también debe tomarse en cuenta. Por ejemplo, el hecho de tener una alta proporción de encuestas no contestadas, ya que las exbeneficiarias no pudieron ser encontradas en los domicilios que ellas proporcionaron (alta movilidad geográfica de personas). En muchas comunidades rurales y urbanas de los municipios donde se aplicaron las encuestas, como Tecomán y Manzanillo, existe una población flotante que nadie conoce (algunos pueden ser familias de jornaleros agrícolas, que se movilizan conforme las temporadas de cosecha de frutas y hortalizas), lo cual dificultó su ubicación durante el trabajo de campo.

También se aclara que los resultados son aplicables a las localidades urbanas y rurales del estado donde Liconsa vende leche líquida, y no en las zonas rurales, donde se venden leche en polvo. Como en estos casos no existe horario y las personas de la comunidad se conocen, no se percibe inseguridad, al menos para comprar la leche de Liconsa. 


\section{Biblografía}

Barrientos, Armando y David Hulme (2009), "Social Protection for the Poor and Poorest in Developing Countries: Reflections on a Quiet Revolution: Commentary", Oxford Development Studies 37(4), pp. 439-456.

Bravo Flores, Roberto (2019), "Estudio de la satisfacción del Programa de Abasto Social de Leche Liconsa en Colima 2018”, Tesis de Maestría en Gestión del Desarrollo, Facultad de Economía, Universidad de Colima, México.

Cogco, Adolfo, Jorge Pérez y Oscar Martínez (2013), "Satisfacción de los programas sociales. El caso del programa de abasto de leche Liconsa", Revista de Economía del Rosario 16(1), pp. 125-147.

Field, Andy (2009), Discovering statistics using IBM SPSS Statistics, Sage Publications, California, EUA.

González-Tiburcio, E. (2001), “La evaluación de programas sociales; aspectos conceptuales y prácticos”, Conferencia, Facultad de Economía, UNAM, México. <https://bit.ly/34hwH1o>. [10 de febrero de 2019].

Greene, William (2012), Econometric Analysis, 7a edición, Prentice Hall, Nueva York, EUA.

Grijalvo-Haro, María Isabel, Elsa Yolanda Chavarría, Elizabeth Artalejo, Amparo Nieblas, José Antonio Ponce y Alma E. Robles-Sardín (2014), “Efecto de la leche fortificada Liconsa en el estado de hierro y zinc en preescolares mexicanos", Nutrición Hospitalaria, 29(2), pp. 331-336.

Hair, Joseph F, Ronald L. Tatham, Rolph E. Anderson y William Black (1999), Análisis multivariante, 5a edición, Prentice Hall Iberia, Madrid, España.

Infante, Said y Guillermo P. Zárate de Lara (2000), Métodos estadísticos. Un enfoque interdisciplinario, Editorial Trillas, Ciudad de México, México.

Kelly, Janet M. y David Swindell (2002), “A multiple-indicator approach to municipal service evaluation: Correlating performance measurement and citizen satisfaction across jurisdictions", Public Administration Review, 62(5), pp. 610-621.

León-Flores, Christian Mario (2010), “Implantación del índice mexicano de satisfacción del usuario en el Programa de Abasto Social de Leche Liconsa y el Programa 70 y más a cargo de la Secretaría de Desarrollo Social”, Tesis de Maestría en Ingeniería de Calidad, Universidad Iberoamericana, Ciudad de México, México.

Lobato-Calleros, Odette, Humberto Rivera-Navarro, Hugo Serrato-González, María Elena Gómez-Cruz y Dominique Brun-Battitini (2016), El desarrollo de una metodología para evaluar la satisfacción de los usuarios de programas sociales en México. El índice mexicano de satisfacción del usuario, Universidad Iberoamericana, Ciudad de México, México.

Montesinos-López, Osval A., Ignacio Luna-Espinoza, Carlos M. Hernández- 
Suárez y Miguel A. Tinoco-Zermeño (2009), Muestreo estadístico. Tamaño de muestra y estimación de parámetros, Universidad de Colima, México.

Muthén, Bengt y David Kaplan (1992), “A comparison of some methodologies for the factor analysis of non-normal Likert variables: A note on the size of the model, British Journal of Mathematical and Statistical Psychology, 38, 171-189.

Santos Peña, Julián, Ángel Muñoz Alamillos, Pedro Juez Marte y Pedro Cortiñas Vázquez (2004), Diseño de encuestas para estudios de mercado. Técnicas de muestreo y análisis multivariante, Editorial Centro de Estudios Ramón Areces, Madrid, España.

Shamah, Teresa, Salvador Villalpando, Verónica Mundo, Lucía Cuevas y Juan Rivera (2007), "Lecciones aprendidas en la evaluación de Liconsa", Salud Pública de México, 49, pp. E250-E254.

Soto-Romero, Jorge Mario et al. (2004), "Evaluación de Resultados del Programa de Abasto Social de Leche, a cargo de Liconsa, S.A. de C.V. Reporte final del periodo enero-diciembre de 2003", Centro de Estudios Estratégicos, Instituto Tecnológico de Estudios Superiores de Monterrey, Ciudad de México, México.

Winkelmann, R. (2011), "Copula bivariate probit models: with an application to medical expenditures", Health Economics, 21(12), pp. 1444-1455. 